\title{
Approaches for Tracking Clouds from Geostationary Satellite Images: A Literature Survey
}

\author{
Chitra Merin Varghese \\ Dept. of Computer Science \\ College of Engineering Poonjar
}

\author{
Sreekumar K. \\ Dept. of Computer Science \\ College of Engineering Poonjar
}

\begin{abstract}
Rainfall and solar irradiance are the two important factors determining the agricultural productivity, availability of drinking water and weather conditions of a region. Rainfall predictions are important to understand manage the utilization of water, as the saying "without rain nothing grows". A sudden storm or a cyclone can cause severe damages to crops and can endanger life of people. For people relying on fishing in the seas as a living hood an unpredicted cyclone or storm can be life threatening. The above mentioned natural phenomena are all predictable by analyzing the clouds in the regions atmosphere. The study of clouds, where they occur and their characteristics, play a key role in understanding of climatic changes. We have a number of geostationary satellites like KALPANA, INSAT etc, orbiting around the earth surface to monitor the Atmospheric Motion Vectors (AMV's) and the optical flow of clouds. Cloud analysis using image processing techniques on satellite images is widely used to predict rainfall availability, cyclone, storm etc. Unlike traditional prediction methods that includes consideration of other climatic factors such as temperature, pressure, humidity etc which involves heavy calculations and speculations, cloud data analysis make the whole process simple and automated. This paper is a survey of different techniques that are deployed over satellite images in order to detect direction of clouds is studied and an evaluation of the accuracy of these methods is done.
\end{abstract}

\section{Keywords}

Cloud Tracking, Weather Prediction, Atmospheric Motion Vector (AMV), Geostationary Satellite, Optical Flow, Remote Sensing, Satellite Image Analysis.

\section{INTRODUCTION}

The earth's climate system constantly adjusts. The study of occurrence and characteristics clouds, play a key role in understanding climatic changes. Various methods have been practiced for weather predictions since early times. As the climate of a region depends on physical properties such as temperature, pressure, humidity etc, traditional prediction methods involved considering these numerical readings for calculations. Traditional techniques are effective, but not good enough to be automated as it requires an expertise person to manually gather the readings. The accuracy of these methods greatly depends on the knowledge and experience of person handling it.

Accurate weather predictions are important for planning dayto-day activities. Farmers and cultivators need information about these climatic factors to plan for the planting and harvesting of their crops. Airlines need to know local weather conditions to schedule flights and ensure its safety. Weather forecasting helps us to be more informed to take daily decisions, and may even help us keep out of danger.
Rain is a natural process of precipitation of water from clouds as a result of what we call, "the Water Cycle". The rainy season, is when the maximum rainfall occurs. The phenomenon of rainfall, which is a part of nature's work flow has now been drastically varying due to several hazardous changes happening to the atmosphere such as global warming, weather shifting, pollution etc. Hence it has become difficult to predict it. It is important to know the time and amount of rainfall as it is the major source of water for various purposes like agriculture, drinking water and other purposes.

Solar irradiance is the energy received from the sun as electro magnetic radiations. It affects plant metabolism and animal behavior. Variations of irradiance happen due to atmospheric absorptions, cloud cover and scattering. Cloud cover affects both the amount of surface heat that is reflected back to space at night and the amount of direct solar energy reaching the surface of earth in the day time. Cloud motion estimation is needed to predict solar irradiance.

Cyclone is a natural phenomenon in which strong wind rotating inwards to a low pressure area with anti clockwise or clockwise circulation. Cyclone is characterized by heavy formation of clouds above the affected area. Meteorologist use modern satellite technologies to track cyclones. Tropical cyclones are difficult to predict, since they suddenly weaken or alter their course. Evaluating the motion, direction and velocity of clouds under such circumstances can help in predicting the course of cyclone.

Geostationary satellite is a satellite orbiting around the earth and is placed directly over the equator and revolves in the same direction that earth rotates. It has the property of remaining fixed in the exact same position in the sky. A sequence of images of earth taken from these satellites can show the movement of cloud and hence allows forecasters to monitor the progress of weather systems.

India has deployed about 11 geostationary satellites. Currently there are 3 meteorological satellites KALPANA-1, INSAT-3A and INSAT-3D in the orbiting above the South Asian sector. The INSAT series that carry Very High Resolution Radiometer (VHRR) have been providing data for generating Cloud Motion Vector (CMV), cloud top temperature, Water Vapor Features (WVF) etc facilitating rainfall estimation, weather forecasting, genesis of cyclones and their tracks prediction.

Image Processing techniques are employed to capture cloud features. Atmospheric Motion Vectors (AMV's), which is a combination of Cloud Motion Vector (CMV) and Water Vapor Features (WVF) can be extracted from the satellite images.

In this paper, different techniques that are deployed over satellite images in order to detect the motion, direction and 
velocity of cloud is studied and an evaluation of these methods is carried out.

\section{REVIEW OF LITERATURE}

Many atmospheric weather conditions like rain, wind, cyclone, solar irradiance can be understood and estimated by doing a detailed study of cloud features. There are a number of prediction methods that had been utilized to understand weather conditions since early times. Initial prediction methods involved direct observation of clouds by expert persons also considering other dependant factors such as temperature, pressure, humidity, seasons etc to determine whether there is a possibility for rain, or a cyclone etc.

Atmospheric Motion Vectors (AMV) describes the space and time features of atmosphere ie cloud and water vapor, that are the important components determining various weather conditions. These AMV's helps in determining the motion and direction of clouds [1]. Geostationary satellites are deployed to capture AMV's of a particular region. More than one channel of light ie visible light channel, infrared channel, water vapor channel are used to capture cloud images. The area under coverage of the satellite varies in accordance to the quality of the satellites. They contain Very High Resolution Radiometer(VHRR) to accurately and automatically capture AMV's. Satellites such as KALPANA-1and the INSAT series are deployed over the South Asian region, particularly India. It captures images within an interval of 30 minutes. Hence they can forecast short climatic variances. [2]

Nowadays, cloud detection and analysis using satellite images are widely used for weather predictions. It is the measurement of irradiance of light coming from the sun that is taken into account for detecting clouds in the satellite images. The more the density of the cloud the less will be irradiance of light and the brighter will it appear in the images. Many methods are used to detect the solar irradiance to forecast the amount of solar energy that reaches the earth's surface. As proposed in [3] (Chang Ki Kim et al.,2016), that aims at the estimation of downwelling surface shortwave radiation by observing satellite images over a certain region in South-Western US for a period of time. A combination of visible channel image and the corresponding IR channel images from satellites were used to differentiate clouded and clear sky regions.

The paper [4] (J. Alonso-Montesinos and F.J. Batlles, 2015) focused on calculating solar components in all sky conditions. To predict the long term and short term future availability of solar radiation, Cloud Motion Vectors (CMV) were calculated using maximum cross correlation method between two consecutive images. Another approach suggested in (Zibo Dong et al.,2013)[5] uses Exponential Smoothing State Space(ESSS) model to forecast Cloud Cover Index (CCI). Multi-Layer Perceptron model is used to derive solar irradiance based on the forecasted CCI. A different method is proposed in [6] (Antonio T. Lorenzo et al., 2016), which evaluates solar irradiance by deploying a network of sensors into the space. In this, a comparison of clear sky data and past available sky conditions are used as training set for predicting solar energy availability in future.

Another natural phenomenon that is widely estimated using cloud detection is cyclone. The occurrence of cyclone is a hazardous natural calamity; it damages properties and is dangerous for living things. Detection of cyclone and its origin can reduce losses considerably. Jia Liu et al., in 2015 [7] proposed a method to locate the center of cyclone by applying edge detection and density matrix upon infrared images obtained from satellites.

Storm accompanies cyclone, which is as much destructive as a cyclone itself. A visual learning approach is put forward by $Y u$ Zhang et al., in 2016 [8], that searches for visual features such as clouds that appear in "comma" shape. Cloud Motion Vectors (CMV) are abstracted from each image and optical flow is calculated to find the direction of motion. Vortex of storm is identified from both visual features and historic storm records. Mesoscale Convection Systems (MCS) is a severe climatic condition that is associated with heavy rainfall, thunder storm, tornado etc. They are very destructive and causes several casualities to human beings and animals. It also affects air flights safety. Lian Duan et al., in 2014 [9] suggested a MCS cloud tracking system which utilizes a target matching principle where each cloud pixel is compared against a standard value to pass a MCS cloud pixel. It mainly focused on identifying and tracking MCS clouds that could possibly occur in the route of air flights, so as to avoid any destruction that could happen to them.

Bibin Johnson et al., 2017 [10] came up with a new proposal which evaluates cloud clusters to finely detect the motion of internal cloud segments. A large cloud can split into smaller segments due to different factors such as wind or dissolution into vapor etc. these segments may follow different directions of motion compared to the initial motion of the cloud as a whole. The approach suggested here combines dense and sparse cloud motion estimation. It calculates number of cloud clusters, its size and speed as well as their life cycle.

\section{COMPARISON}

We have come across different methods used for various atmospheric predictions. A tabulation of these data will enhance the clarity of our understandings. Table 1 gives the summary of the review.

\section{FINDINGS}

Form a sequence of images it is possible to locate the center of the cloud. The points thus obtained can be fitted into a function. Applying this function into neural network model can help in predicting the path of the moving cloud. Various challenges are faced when it comes to cloud detection from satellite images. In regions where snow or large ice bergs are present, cloud detection may be difficult, misinterpretation leads to erroneous data. Although we use high resolution satellite cameras, there are limitations in detecting thin layers of cloud. Since cloud is not a rigid body, it dissolves into the atmosphere making the analysis more difficult.

\section{CONCLUSION}

There are many methods and techniques in image processing to detect clouds and thereby estimating the direction of their flow, and it's velocity from a satellite image. We see that cloud analysis is used in various types of natural phenomenon predictions such as rainfall, storm, cyclone, solar irradiance etc. We also get a thorough understanding of the various satellites used to capture image data. From the studies we come across the techniques in a way that betters the previous ones used. We see that the comparison table furnished above gives a better understanding of the different techniques used by different authors. 
Table 1. Comparison of different cloud detection techniques using satellite images.

\begin{tabular}{|c|c|c|c|c|}
\hline S.No & Publication & Tracing Method & Data Set & Result Obtained \\
\hline 1. & $\begin{array}{l}\text { Toward Improved Solar } \\
\text { Irradiance } r \text { Forecasts: } \\
\text { Derivation of Downwelling } \\
\text { Surface Shortwave Radiation } \\
\text { in Arizona from Satellite - } \\
\text { 2016. [3] }\end{array}$ & $\begin{array}{l}\text { A combination of visible channel } \\
\text { image and the corresponding IR } \\
\text { channel images from satellites } \\
\text { were used to differentiate clouded } \\
\text { and clear sky regions. }\end{array}$ & $\begin{array}{l}\text { GOES data collected } \\
\text { every } 15 \text { min during } \\
\text { daytime hours (1400- } \\
\text { 0000 UTC) from January } \\
1,2014 \text { to December } 31 \text {, } \\
2014 .\end{array}$ & $\begin{array}{l}\text { Estimated } \\
\text { downwelling } \\
\text { surface shortwave } \\
\text { radiation. }\end{array}$ \\
\hline 2. & $\begin{array}{l}\text { Solar radiation forecasting in } \\
\text { the short- and medium-term } \\
\text { under all sky conditions - } \\
\text { 2015. [4] }\end{array}$ & $\begin{array}{l}\text { Cloud Motion Vectors (CMV) } \\
\text { were calculated using maximum } \\
\text { cross correlation method between } \\
\text { two consecutive images }\end{array}$ & $\begin{array}{l}\text { Satellite data from the } \\
\text { years } 2010-2014 \text { were } \\
\text { used. } 5 \text { MSG satellite } \\
\text { channels were collected, } \\
\text { received every } 15 \text { min. }\end{array}$ & $\begin{array}{l}\text { Predict the long } \\
\text { term and short term } \\
\text { future availability } \\
\text { of solar radiation. }\end{array}$ \\
\hline 3. & $\begin{array}{l}\text { Satellite image analysis and a } \\
\text { hybrid ESSS/ANN model to } \\
\text { forecast solar irradiance in the } \\
\text { tropics - 2013. [5] }\end{array}$ & $\begin{array}{l}\text { Exponential Smoothing State } \\
\text { Space(ESSS) model. } \\
\text { Multi-Layer Perceptron model. }\end{array}$ & $\begin{array}{ll}\text { Multifunctional } & \\
\text { Transport } & \\
\text { Satellites (MTSAT) } & 2, \\
\text { also known as } & \text { as } \\
\text { Himawari-7 } & \end{array}$ & $\begin{array}{l}\text { Forecasted hourly } \\
\text { resolution solar } \\
\text { irradiance data }\end{array}$ \\
\hline 4. & $\begin{array}{l}\text { Irradiance Forecasts based on } \\
\text { an Irradiance Monitoring } \\
\text { Network, Cloud Motion, and } \\
\text { Spatial Averaging - 2016. [6] }\end{array}$ & $\begin{array}{l}\text { Trained data set was used to } \\
\text { predict the future irradiance. } \\
\text { Collected images were compared } \\
\text { against the trained dataset. }\end{array}$ & $\begin{array}{l}\text { Deployed a network of } \\
\text { sensors into the space. } \\
\text { Collected images for a } \\
\text { period of time. }\end{array}$ & $\begin{array}{l}\text { Improved very } \\
\text { short time horizon } \\
\text { forecasts }\end{array}$ \\
\hline 5. & $\begin{array}{l}\text { A Novel Algorithm For } \\
\text { Detecting Center Of Tropical } \\
\text { Cyclone In Satellite Infrared } \\
\text { Image-2015. [7] }\end{array}$ & $\begin{array}{l}\text { Edge detection and Density matrix } \\
\text { upon infrared images. }\end{array}$ & $\begin{array}{l}\text { IR data from FY-2F } \\
\text { (fengyun-2F } \\
\text { geostationary } \\
\text { meteorological satellite) }\end{array}$ & $\begin{array}{l}\text { Detected center of } \\
\text { cyclone } \\
\text { automatically. }\end{array}$ \\
\hline 6. & $\begin{array}{l}\text { Storm Detection by Visual } \\
\text { Learning Using Satellite } \\
\text { Images - 2016. [8] }\end{array}$ & $\begin{array}{l}\text { Cloud Motion Vectors (CMV) } \\
\text { were abstracted. } \\
\text { Optical flow of cloud was } \\
\text { estimated. }\end{array}$ & $\begin{array}{l}\text { Images form } \\
\text { Geostationary } \\
\text { Operational } \\
\text { Environmental } \\
\text { Satellite(GOES) system } \\
\text { of the year 2008. }\end{array}$ & $\begin{array}{l}\text { Vortex of storm } \\
\text { was identified. }\end{array}$ \\
\hline 7. & $\begin{array}{l}\text { Research on Automatic } \\
\text { Tracking of MCS Based on } \\
\text { Infrared Satellite Cloud } \\
\text { Image of FY2D - 2014. [9] }\end{array}$ & Target matching principle. & $\begin{array}{l}\text { Infrared Image of } \\
\text { (Feng-Yun - 2D) FY2D } \\
\text { Satellite. }\end{array}$ & $\begin{array}{l}\text { Automatic } \\
\text { identification } \\
\text { MCS cloud. }\end{array}$ \\
\hline 8. & $\begin{array}{l}\text { A Novel Visualization and } \\
\text { Tracking Framework for } \\
\text { Analyzing the Inter/Intra } \\
\text { Cloud Pattern Formation to } \\
\text { Study Their Impact on } \\
\text { Climate - 2017. [10] }\end{array}$ & $\begin{array}{l}\text { Combines dense and sparse cloud } \\
\text { motion estimation. } \\
\text { Region overlap. } \\
\text { Registration, preprocessing, } \\
\text { segmentation, and tracking. }\end{array}$ & $\begin{array}{l}\text { KALPANA-1 satellite } \\
\text { images. }\end{array}$ & $\begin{array}{l}\text { Better estimation of } \\
\text { direction of motion } \\
\text { of clouds. }\end{array}$ \\
\hline
\end{tabular}




\section{REFERENCES}

[1] Matthew A. Lazzara and Jeffrey R. Key, High-Latitude Atmospheric Motion Vectors from Composite Satellite Data. Journal Of Applied Meteorology And Climatology, 2013, Volume 53.

[2] Vijay Garg and R.K. Giri, Atmospheric Motion Vectors (AMVs) and their forecasting significance. International Journal of Engineering Research and Management Technology.ISSN:23484039.Volume-1, Issue-1, 2014.

[3] Chang Ki Kim, William F. Holmgren, Michael Stovern, and Eric A. Betterton, Toward Improved Solar Irradiance Forecasts: Derivation of Downwelling Surface Shortwave Radiation in Arizona from Satellite. Pure and Applied Geophysic, Springer International Publishing, 2016.

[4] J. Alonso-Montesinos and F.J. Batlles, Solar radiation forecasting in the short- and medium-term under all sky conditions. Energy 83 (2015) 387e393 Elsevier.

[5] Zibo Dong, Dazhi Yang, Thomas Reindl, Wilfred M. Walsh, Satellite image analysis and a hybrid ESSS/ANN model to forecast solar irradiance in the tropics. Energy Conversion and Management 79 (2014) 66-73. Elsevier, 2013
[6] Antonio T. Lorenzoa, William F. Holmgrenb, Alexander D. Croninc, Irradiance Forecasts based on an Irradiance Monitoring Network, Cloud Motion, and Spatial Averaging. j.solener, 2016.

[7] Jia Liu, Chuancai Liu, Boyang Wang, Danyu Qin, A Novel Algorithm For Detecting Center Of Tropical Cyclone In Satellite Infrared Image. The International Geoscience and Remote Sensing Symposium, 2015.

[8] Yu Zhang, Stephen Wistar, Jia Li, Michael Steinberg and James Z. Wan, Storm Detection by Visual Learning Using Satellite Images. arXiv:1603.00146v1 [cs.CV], 2016.

[9] Lian Duan, Junjie Wu and Fan Liu, Research on Automatic Tracking of MCS Based on Infrared Satellite Cloud Image of FY2D. Applied Mechanics and Materials Vols 716-717 (2015) pp 1089-1092.Trans Tech Publications.

[10] Bibin Johnson, J.Sheeba Rani and Gorthi R.K.S.S Manyam, 2017. A Novel Visualization and Tracking Framework for Analyzing the Inter/Intra Cloud Pattern Formation to Study Their Impact on Climate. Proceedings of International Conference on Computer Vision and Image Processing, Advances in Intelligent [11] 